\title{
On Unitary Invariant Strongly Pseudoconvex Complex Landsberg Metrics
}

\author{
Yong $\mathrm{He}^{1,2}$, Chun-Ping Zhong ${ }^{1, *}$ \\ ${ }^{1}$ School of Mathematical Sciences, Xiamen University, Xiamen 361005, Fujian, \\ P.R. China. \\ ${ }^{2}$ School of Mathematical Sciences, Xinjiang Normal University, Urumqi 830017, \\ Xinjiang, P.R. China.
}

Received 21 December 2015; Accepted 22 Febuary 2016

\begin{abstract}
In this paper, we prove that a unitary invariant strongly pseudoconvex complex Finsler metric is a complex Landsberg metric if and if only if it comes from a unitary invariant Hermitian metric. This implies that there does not exist unitary invariant complex Landsberg metric unless it comes from a unitary invariant Hermitian metric.
\end{abstract}

AMS subject classifications: 53C60, 53C40.

Key words: Unitary invariant, complex Landsberg metric, complex Finsler metric.

\section{Introduction}

In real Finsler geometry, a Berwald metric is necessary a Landsberg metric. It is still an open problem that whether there exists a Landsberg metric which does not come from a Berwald metric [2]. This problem is also called the unicorn problem by D. Bao [3] and M. Matsumoto [2].

M. Matsumoto conjectured that there does not exist unicorn metric, which implies that every Landsberg metric comes from a Berwald metric. In 2008, Z. I. Szabó claimed that all regular Landsberg metrics are Berwald metrics [4]. A gap, however, was soon found in the proof by himself [5], thus leaving the problem still open.

On the other hand, in $[6,7]$, G. S. Asnov constructed a family of almost regular unicorn metrics which come from $(\alpha, \beta)$-metrics. In 2009, Z. Shen [8] characterized almost regular Landsberg $(\alpha, \beta)$-metrics which generalized G. S. Asanovs results. For the spherically symmetric real Finsler metrics which are not necessary $(\alpha, \beta)$-metrics, X.-H. Mo and L.-F. Zhou [9] proved that there does not exist any non-Berwaldian Landsberg metrics among the regular case.

${ }^{*}$ Corresponding author. Email addresses: heyong@xjnu.edu. cn (Y. He), zcp@xmu.edu.cn. (C.P. Zhong) 
In complex Finsler geometry, there are also notions of complex Berwald metric, weakly complex Berwald metric, and complex Landsberg metric. It was known that every KählerBerwald metric is necessary a complex Landsberg metric [10]. One may wonder whether there exists a complex Landsberg metric which does not come from a Kähler-Berwald metric?

Unlike in real Finsler geometry, there are few explicit examples of strongly pseudoconvex complex Finsler metrics in literatures. This situation has already been changed because of the recent work of C.-P. Zhong [12], where unitary invariant strongly pseduoconvex complex Finsler metrics were systematically studied and the explicit method of constructing strongly psuedoconvex or even strongly convex complex Finsler metrics were given. In [13], H.C. Xia and C.-P. Zhong gave a classification of unitary invariant weakly complex Berwald metrics which are of constant holomorphic curvatures. It was proved in [12] that there is neither complex Berwald metric nor Kähler Finsler metric which is unitary invariant and does not come from a Hermitian metric. There are, however, lots of weakly complex Berwald metrics which are unitary invariant and they do not come from Hermitian metrics. One may wonder whether there exists unitary invariant complex Landsberg metric which does not come from a Kähler-Berwald metric.

In this paper, we prove that a unitary invariant strongly pseudoconvex complex Finsler metric is a complex Landsberg metric if and only if it comes from a unitary invariant Hermitian metric. This implies that there does not exist unitary invariant complex Landsberg metric unless it comes from a unitary invariant Hermitian metric.

\section{Preliminary}

Let $\mathbb{C}^{n}$ be a complex $n$ dimensional linear space, denote by $\langle\cdot, \cdot\rangle$ the canonical complex Euclidean inner product and $\|\cdot\|$ the induced norm in $\mathbb{C}^{n}$. Let $F$ be a strongly pseudoconvex complex Finsler metric on a unitary invariant domain $D \subset \mathbb{C}^{n}$. It was proved in [12] that $F$ is unitary invariant if and only if there exists a smooth function $\phi(t, s):[0,+\infty] \times[0,+\infty] \rightarrow(0,+\infty)$ such that $F=\sqrt{r \phi(t, s)}$ with

$$
r=\|v\|^{2}, t=\|z\|^{2}, s=\frac{|\langle z, v\rangle|^{2}}{\|v\|^{2}},
$$

where $z=\left(z^{1}, \cdots, z^{n}\right) \in D$ and $v=\left(v^{1}, \cdots, v^{n}\right) \in T_{z}^{1,0} D$.

Lemma 2.1. [12] Let $F=\sqrt{r \phi(t, s)}$ be a strongly pseudoconvex complex Finsler metrics defined on a domain $D \subset \mathbb{C}^{n}$. Then the fundamental tensor of $F$ is

$$
G_{\gamma \bar{\tau}}=\left(\phi-s \phi_{s}\right) \delta_{\gamma \bar{\tau}}+r \phi_{s s} s_{\gamma} s_{\bar{\tau}}+\phi_{s} \overline{z^{\gamma}} z^{\tau} .
$$

It is known that for a strongly pseudoconvex complex Finsler metric $F$, there are several complex Finsler connections associated to it. The most often used complex Finsler connections are the Chern-Finsler connection [1], the complex Rund connection and the 
complex Berwald connection [11]. These connections are convenient respectively in considering different type of problems in complex Finsler geometry. In this paper, we need the complex Berwald connection associated to $F$. The connection 1-forms of complex Berwald connection are given by $\breve{\omega}_{\beta}^{\alpha}=\mathbb{G}_{\beta \mu}^{\alpha} d z^{\mu}$, where

$$
\mathbb{G}_{\beta \mu}^{\alpha}=\dot{\partial}_{\beta}\left(\mathbb{G}_{\mu}^{\alpha}\right), \quad \mathbb{G}_{\mu}^{\gamma}=\dot{\partial}_{\mu}\left(\mathbb{G}^{\gamma}\right)
$$

Here $\mathbb{G}^{\gamma}=\frac{1}{2} \Gamma_{; \mu}^{\gamma} v^{\mu}$ are the complex geodesic spray coefficients associated to $F$, and $\Gamma_{; \mu}^{\gamma}=$ $G^{\bar{\beta} \gamma} G_{\bar{\beta} ; \mu}$ are the Chern-Finsler nonlinear coefficients associated to $F$.

Lemma 2.2. [12] Let $F=\sqrt{r \phi(t, s)}$ be a strongly pseudoconvex complex Finsler metrics defined on a domain $D \subset \mathbb{C}^{n}$. Then the complex Berwald nonlinear connection coefficients $\mathbb{G}_{v}^{\gamma}$ associated to $F$ are given by

$$
2 \mathbf{G}_{v}^{\gamma}=n_{1} \overline{z^{v}} v^{\gamma}-\frac{s}{r} \frac{\partial k_{2}}{\partial s} \overline{\langle z, v\rangle} \overline{v^{v}} v^{\gamma}+k_{2} \overline{\langle z, v\rangle} \delta_{v}^{\gamma}+\left(m_{1}+k_{3}\right) \overline{\langle z, v\rangle} \overline{z^{v}} z^{\gamma}-\frac{s}{r} \frac{\partial k_{3}}{\partial s}(\overline{\langle z, v\rangle})^{2} \overline{v^{v}} z^{\gamma},
$$

where

$$
\begin{aligned}
& n_{1}=s \frac{\partial k_{2}}{\partial s}+k_{2}, \quad k_{1}=\left(\phi-\phi_{s}\right)\left[\phi+(t-s) \phi_{s}\right]+s(t-s) \phi \phi_{s s}, \\
& k_{2}=\frac{1}{k_{1}}\left\{\left[\phi+(t-s) \phi_{s}+s(t-s) \phi_{s s}\right]\left(\phi_{t}+\phi_{s}\right)-s\left[\phi+(t-s) \phi_{s}\right]\left(\phi_{s t}+\phi_{s s}\right)\right\}, \\
& k_{3}=\frac{1}{k_{1}}\left\{\phi\left(\phi_{s t}+\phi_{s s}\right)-\phi_{s}\left(\phi_{t}+\phi_{s}\right)\right\} \\
& m_{1}=s \frac{\partial k_{3}}{\partial s}+k_{3} .
\end{aligned}
$$

Lemma 2.3. Let $F=\sqrt{r \phi(t, s)}$ be a strongly pseudoconvex complex Finsler metric defined on a domain $D \subset \mathbb{C}^{n}$. Then the complex Berwald connection coefficients $\mathbb{G}_{v \mu}^{\gamma}$ are given by

$$
\begin{aligned}
& 2 \mathbb{G}_{v \mu}^{\gamma}=\frac{s}{r^{2}} m_{2}(\overline{\langle z, v\rangle})^{2} \overline{v^{\mu} v^{v}} z^{\gamma}-\frac{s}{r} m_{3} \overline{\langle z, v\rangle} \overline{z^{\mu} v^{v}} z^{\gamma}-\frac{s}{r} m_{3} \overline{\langle z, v\rangle} \overline{z^{v} v^{\mu}} z^{\gamma} \\
& +\frac{s}{r^{2}} n_{2} \overline{\langle z, v\rangle} \overline{v^{\mu} v^{v}} v^{\gamma}-\frac{s}{r} \frac{\partial k_{2}}{\partial s} \overline{\langle z, v\rangle} \overline{v^{v}} \delta_{\mu}^{\gamma}-\frac{s}{r} \frac{\partial k_{2}}{\partial s} \overline{\langle z, v\rangle} \overline{v^{\mu}} \delta_{v}^{\gamma} \\
& +\frac{1}{r} n_{2}\langle z, v\rangle \overline{z^{\mu} z^{v}} v^{\gamma}+\left(s m_{3}+m_{1}+k_{3}\right) \overline{z^{\mu} z^{v}} z^{\gamma}-\frac{s}{r} n_{2} \overline{z^{\mu} v^{v}} v^{\gamma} \\
& -\frac{s}{r} n_{2} \overline{z^{v} v^{\mu}} v^{\gamma}+n_{1} \delta_{v}^{\gamma} \overline{z^{\mu}}+n_{1} \delta_{\mu}^{\gamma} \overline{z^{v}}
\end{aligned}
$$

where

$$
m_{2}=s \frac{\partial^{2} k_{3}}{\partial s^{2}}+2 \frac{\partial k_{3}}{\partial s}, \quad m_{3}=s \frac{\partial^{2} k_{3}}{\partial s^{2}}+3 \frac{\partial k_{3}}{\partial s}, \quad n_{2}=s \frac{\partial^{2} k_{2}}{\partial s^{2}}+2 \frac{\partial k_{2}}{\partial s}
$$


Proof. Note that

$$
\frac{\partial n_{1}}{\partial v^{\mu}}=\left(s \frac{\partial^{2} k_{2}}{\partial s^{2}}+2 \frac{\partial k_{2}}{\partial s}\right) s_{\mu}, \quad \frac{\partial m_{1}}{\partial v^{\mu}}=\left(s \frac{\partial^{2} k_{3}}{\partial s^{2}}+2 \frac{\partial k_{3}}{\partial s}\right) s_{\mu}
$$

Thus differentiating (2.2) with respect to $v^{v}$, we get

$$
\begin{aligned}
2 \mathbb{G}_{v \mu}^{\gamma}=2 \dot{\partial}_{\mu} & \left(\mathbb{G}_{v}^{\gamma}\right) \\
=(s & \left.\frac{\partial^{2} k_{2}}{\partial s^{2}}+2 \frac{\partial k_{2}}{\partial s}\right) s_{\mu} \overline{z^{v}} v^{\gamma}+n_{1} \overline{z^{v}} \delta_{\mu}^{\gamma}-\frac{1}{r} \frac{\partial k_{2}}{\partial s} \overline{\langle z, v\rangle} s_{\mu} \overline{v^{v}} v^{\gamma} \\
& +\frac{s}{r^{2}} \frac{\partial k_{2}}{\partial s} \overline{\langle z, v\rangle} \overline{v^{\mu} v^{v}} v^{\gamma}-\frac{s}{r} \frac{\partial^{2} k_{2}}{\partial s^{2}} \overline{\langle z, v\rangle} s_{\mu} \overline{v^{v}} v^{\gamma}-\frac{s}{r} \frac{\partial k_{2}}{\partial s} \overline{z^{\mu} v^{v}} v^{\gamma} \\
& -\frac{s}{r} \frac{\partial k_{2}}{\partial s} \overline{\langle z, v\rangle} \overline{v^{v}} \delta_{\mu}^{\gamma}+\frac{\partial k_{2}}{\partial s} s_{\mu} \overline{\langle z, v\rangle} \delta_{v}^{\gamma}+k_{2} \overline{z^{\mu}} \delta_{v}^{\gamma} \\
& +\left(s \frac{\partial^{2} k_{3}}{\partial s^{2}}+3 \frac{\partial k_{3}}{\partial s}\right) \overline{\langle z, v\rangle} s_{\mu} \overline{z^{v}} z^{\gamma}+\left(m_{1}+k_{3}\right) \overline{z^{\mu} z^{v}} z^{\gamma} \\
& -\frac{1}{r} \frac{\partial k_{3}}{\partial s}(\overline{\langle z, v\rangle})^{2} s_{\mu} \overline{v^{v}} z^{\gamma}+\frac{s}{r^{2}} \frac{\partial k_{3}}{\partial s}(\overline{\langle z, v\rangle})^{2} \overline{v^{\mu} v^{v}} z^{\gamma} \\
& -\frac{s}{r} \frac{\partial^{2} k_{3}}{\partial s^{2}}(\overline{\langle z, v\rangle})^{2} s_{\mu} \overline{v^{v}} z^{\gamma}-\frac{2 s}{r} \frac{\partial k_{3}}{\partial s} \overline{\langle z, v\rangle \overline{z^{\mu} v^{v}} z^{\gamma}},
\end{aligned}
$$

from which we have

$$
\begin{aligned}
& 2 \mathbb{G}_{v \mu}^{\gamma}=\frac{1}{r}\left(s \frac{\partial^{2} k_{2}}{\partial s^{2}}+2 \frac{\partial k_{2}}{\partial s}\right)\langle z, v\rangle \overline{z^{\mu} z^{v}} v^{\gamma}-\frac{s}{r}\left(s \frac{\partial^{2} k_{2}}{\partial s^{2}}+2 \frac{\partial k_{2}}{\partial s}\right) \overline{z^{v} v^{\mu}} v^{\gamma} \\
& +n_{1} \overline{z^{v}} \delta_{\mu}^{\gamma}-\frac{s}{r} \frac{\partial k_{2}}{\partial s} \overline{z^{\mu} v^{v}} v^{\gamma}+\frac{s}{r^{2}} \frac{\partial k_{2}}{\partial s} \overline{\langle z, v\rangle} \overline{v^{\mu} v^{v}} v^{\gamma}
\end{aligned}
$$

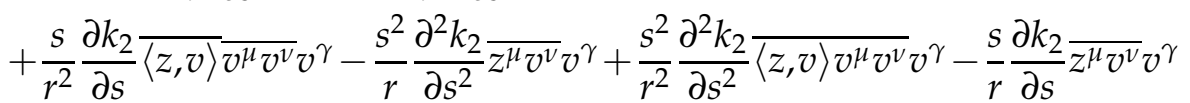

$$
\begin{aligned}
& -\frac{s}{r} \frac{\partial k_{2}}{\partial s} \overline{\langle z, v\rangle} \overline{v^{v}} \delta_{\mu}^{\gamma}+s \frac{\partial k_{2}}{\partial s} \overline{z^{\mu}} \delta_{v}^{\gamma}-\frac{s}{r} \frac{\partial k_{2}}{\partial s} \overline{\langle z, v\rangle} \overline{v^{\mu}} \delta_{v}^{\gamma}+k_{2} \overline{z^{\mu}} \delta_{v}^{\gamma} \\
& +s\left(s \frac{\partial^{2} k_{3}}{\partial s^{2}}+3 \frac{\partial k_{3}}{\partial s}\right) \overline{z^{\mu} z^{v}} z^{\gamma}-\frac{s}{r}\left(s \frac{\partial^{2} k_{3}}{\partial s^{2}}+3 \frac{\partial k_{3}}{\partial s}\right) \overline{\langle z, v\rangle} \overline{z^{v} v^{\mu}} z^{\gamma}+\left(m_{1}+k_{3}\right) \overline{z^{\mu} z^{v}} z^{\gamma} \\
& -\frac{s}{r} \frac{\partial k_{3}}{\partial s} \overline{\langle z, v\rangle} \overline{z^{\mu} v^{v}} z^{\gamma}+\frac{s}{r^{2}} \frac{\partial k_{3}}{\partial s}(\overline{\langle z, v\rangle})^{2} \overline{v^{\mu} v^{v}} z^{\gamma}+\frac{s}{r^{2}} \frac{\partial k_{3}}{\partial s}(\overline{\langle z, v\rangle})^{2} \overline{v^{\mu} v^{v}} z^{\gamma} \\
& -\frac{s^{2}}{r} \frac{\partial^{2} k_{3}}{\partial s^{2}} \overline{\langle z, v\rangle} \overline{z^{\mu} v^{v}} z^{\gamma}+\frac{s^{2}}{r^{2}} \frac{\partial^{2} k_{3}}{\partial s^{2}}(\overline{\langle z, v\rangle})^{2} \overline{v^{\mu} v^{v}} z^{\gamma}-\frac{2 s}{r} \frac{\partial k_{3}}{\partial s} \overline{\langle z, v\rangle} \overline{z^{\mu} v^{v}} z^{\gamma} .
\end{aligned}
$$

The lemma is completed by rearranging terms in the above equality.

Lemma 2.4. Let $F=\sqrt{r \phi(t, s)}$ be a strongly pseudoconvex complex Finsler metric defined on a 
domain $D \subset \mathbb{C}^{n}$. Then the complex Cartan tensor $G_{\mu \bar{\tau} \alpha}$ and $G_{\mu \bar{\tau} ; \nu}$ are given respectively by

$$
\begin{aligned}
G_{\mu \bar{\tau} \alpha}= & {\left[-s \phi_{s s} \delta_{\mu \bar{\tau}}-\frac{1}{r}\left(\phi_{s s}+s \phi_{s s s}\right) \overline{\langle z, v\rangle} \overline{v^{\mu}} z^{\tau}+\frac{s}{r}\left(2 \phi_{s s}+s \phi_{s s s}\right) \overline{v^{\mu}} v^{\tau}\right.} \\
& \left.-\frac{1}{r}\left(\phi_{s s}+s \phi_{s s s}\right)\langle z, v\rangle \overline{z^{\mu}} v^{\tau}+\left(\phi_{s s}+s \phi_{s s s}\right) \overline{z^{\mu}} z^{\tau}\right] s_{\alpha}+\frac{1}{r} \phi_{s s}\langle z, v\rangle \overline{z^{\mu} z^{\alpha} z^{\tau}} \\
& -\frac{s}{r} \phi_{s s} \overline{z^{\alpha} v^{\mu}} z^{\tau}-\frac{s}{r} \phi_{s s} \overline{z^{\mu} v^{\alpha}} z^{\tau}+\frac{s}{r^{2}} \phi_{s s}\langle z, v\rangle \overline{z^{\mu} v^{\alpha}} v^{\tau}+\frac{s}{r^{2}} \phi_{s s} \overline{\langle z, v\rangle \overline{v^{\mu} v^{\alpha}} z^{\tau}} \\
& -\frac{s^{2}}{r^{2}} \phi_{s s} \overline{v^{\mu} v^{\alpha} v^{\tau}}-\frac{s}{r} \phi_{s s}\langle z, v\rangle \overline{z^{\mu}} \delta_{\tau \alpha}+\frac{s^{2}}{r} \phi_{s s} \overline{v^{\mu}} \delta_{\tau \alpha},
\end{aligned}
$$

and

$$
\begin{aligned}
& G_{\mu \bar{\tau} ; \nu}=\left(\phi_{s}+s \phi_{s s}\right) \delta_{\tau v} \overline{z^{\mu}}+\left(\phi_{t}-s \phi_{s t}\right) \delta_{\mu \bar{\tau}} \overline{z^{v}}-\frac{s}{r} \phi_{s s} \overline{\langle z, v\rangle} \delta_{\mu \bar{\tau}} \overline{v^{v}}-\frac{s}{r} \phi_{s s} \overline{\langle z, v\rangle} \delta_{v \tau} \overline{v^{\mu}} \\
& +\left(s \phi_{s s t}+\phi_{s t}\right) \overline{z^{\mu} z^{v}} z^{\tau}+\frac{s^{2}}{r} \phi_{s s t} \overline{v^{\mu} z^{v}} v^{\tau}-\frac{s}{r}\left(s \phi_{s s s}+2 \phi_{s s}\right) \overline{z^{\mu} v^{v}} v^{\tau} \\
& -\frac{s}{r} \phi_{s s t}\langle z, v\rangle \overline{z^{\mu} z^{v}} v^{\tau}-\frac{s}{r} \phi_{s s t} \overline{\langle z, v\rangle} \overline{v^{\mu} z^{v}} z^{\tau}+\frac{1}{r}\left(s \phi_{s s s}+2 \phi_{s s}\right) \overline{\langle z, v\rangle} \overline{z^{\mu} v^{v}} z^{\tau} \\
& +\frac{s}{r^{2}}\left(s \phi_{s S S}+2 \phi_{S S}\right) \overline{\langle z, v\rangle} \overline{v^{\mu} v^{v}} v^{\tau}-\frac{1}{r^{2}}\left(s \phi_{s S S}+\phi_{S S}\right)(\overline{\langle z, v\rangle})^{2} \overline{v^{\mu} v^{v}} z^{\tau} \text {. }
\end{aligned}
$$

Proof. By a simple calculation, we have

$$
\begin{aligned}
& s_{\mu}=\frac{1}{r}\left[\langle z, v\rangle \overline{z^{\mu}}-s \overline{v^{\mu}}\right], s_{\mu \alpha}=-\frac{1}{r}\left(s_{\mu} \overline{v^{\alpha}}+s_{\alpha} \overline{v^{\mu}}\right), \\
& s_{\bar{\tau}}=\frac{1}{r}\left[\overline{\langle z, v\rangle} z^{\tau}-s v^{\tau}\right], s_{\bar{\tau} \alpha}=\frac{1}{r}\left(\overline{z^{\alpha}} z^{\tau}-s_{\alpha} v^{\tau}-s_{\bar{\tau}} \overline{v^{\alpha}}-s \delta_{\tau \alpha}\right), \\
& s_{; v}=\frac{1}{r} \overline{\langle z, v\rangle} \overline{v^{v}}, s_{\mu ; \nu}=\frac{1}{r}\left[\overline{z^{\mu} v^{v}}-\frac{1}{r} \overline{\langle z, v\rangle} \overline{v^{\mu} v^{v}}\right], \\
& s_{\bar{\tau} ; v}=\frac{1}{r}\left[\overline{\langle z, v\rangle} \delta_{\tau v}-\frac{1}{r} \overline{\langle z, v\rangle} \overline{v^{v}} v^{\tau}\right] .
\end{aligned}
$$

Differentiating (2.1) with respect to $v^{\alpha}$, we get

$$
\begin{aligned}
G_{\mu \bar{\tau} \alpha}= & -s \phi_{s s} \delta_{\mu \bar{\tau} s_{\alpha}}-\phi_{s s} s_{\bar{\tau}} \overline{v^{\mu}} s_{\alpha}+\phi_{s s} \overline{z^{\mu}} z^{\tau} s_{\alpha}+\phi_{s s} \overline{z^{\alpha}} z^{\tau} s_{\mu} \\
& -\phi_{s s} s_{\mu} s_{\alpha} v^{\tau}+r \phi_{s s s} s_{\mu} s_{\bar{\tau}} s_{\alpha}-\phi_{s s} s_{\mu} s_{\bar{\tau}} \overline{v^{\alpha}}-s \phi_{s s} \delta_{\tau \alpha} s_{\mu} .
\end{aligned}
$$

Substituting (2.10)-(2.13) into (2.14), we obtain (2.8).

Next differentiating (2.1) with respect to $z^{v}$, we have

$$
\begin{aligned}
G_{\mu \bar{\tau} ; \nu}= & \left(\phi_{t} \overline{z^{v}}-s \phi_{s t} \overline{z^{v}}-s \phi_{s s} s_{; v}\right) \delta_{\mu \bar{\tau}}+r \phi_{s s t} s_{\mu} s_{\bar{\tau}} \overline{z^{v}}+r \phi_{s s s} s_{\mu} s_{\bar{\tau}} s_{; v} \\
& +r \phi_{s s} s_{\mu ; \nu} s_{\bar{\tau}}+r \phi_{s s} s_{\bar{\tau} ; \nu} s_{\mu}+\phi_{s t} \overline{z^{\mu} z^{v}} z^{\tau}+\phi_{s s} \overline{z^{\mu}} z^{\tau} s_{; v}+\phi_{s} \delta_{\tau v} \overline{z^{\mu}} .
\end{aligned}
$$

Substituting (2.10)-(2.13) into (2.15), we obtain (2.9). 
Theorem 2.1. ([12]) A strongly pseudoconvex complex Finsler metrics $F=\sqrt{r \phi(t, s)}$ defined on a domain $D \subset \mathbb{C}^{n}$ is a complex Berwald metric if and only if

$$
\phi_{s s}=0,
$$

if and only if $\phi(t, s)=a_{0}(t)+a_{1}(t)$ s for smooth real-valued functions $a_{0}(t)$ and $a_{1}(t)$ satisfying $a_{0}(t)>0$ and $a_{0}(t)+t a_{1}(t)>0$.

\section{Complex Landersberg metrics}

Complex Landsberg metric is an analogue notion in complex Finsler geometry, which was introduced by M. Aldea and M. Munteanu in [10]. Denote by $\check{\delta}_{v}=\partial_{v}-\mathbb{G}_{v}^{\alpha} \dot{\partial}_{\alpha}$ for $v=1, \cdots, n$ and define

$$
\mathbb{L}_{v \mu}^{\gamma}=\frac{1}{2} G^{\bar{\tau} \gamma}\left(\check{\delta}_{v} G_{\mu \bar{\tau}}+\check{\delta}_{\mu} G_{v \bar{\tau}}\right) .
$$

A strongly pseudoconvex complex Finsler metric $F$ is called a complex Landsberg metric if $\mathbb{G}_{v \mu}^{\gamma}=\mathbb{L}_{v \mu}^{\gamma}$, where $\mathbb{G}_{v \mu}^{\gamma}$ are the complex Berwald connection coefficients. $\mathbb{L}_{v \mu}^{\gamma}$ are the horizontal connection coefficients of the Rund type complex linear connection in the sense of M. Munteanu [11].

Among unitary invariant strongly pseudoconvex complex Finsler metrics there exists no complex Berwald metric which does not come from a unitary invariant Hermitian metric. One may wonder that among unitary invariant strongly pseudoconvex complex Finsler metrics, whether there exists a complex Landsberg metric which does not come from a unitary invariant Hermitian metric? If there exists such a metric, then it implies that the unicorn metric problem in complex Finsler geometry does not hold.

Theorem 3.1. A strongly pseudoconvex complex Finsler metrics $F=\sqrt{r \phi(t, s)}$ defined on a domain $D \subset \mathbb{C}^{n}$ is a complex Landsberg metric if and only if

$$
\phi_{s s}=0,
$$

if and only if $\phi(t, s)=a_{0}(t)+a_{1}(t)$ s for smooth real-valued functions $a_{0}(t)$ and $a_{1}(t)$ satisfying $a_{0}(t)>0$ and $a_{0}(t)+t a_{1}(t)>0$.

Proof. If $\phi_{s s}=0$, then (2.1) gives

$$
G_{\gamma \bar{\tau}}=\left(\phi-s \phi_{s}\right) \delta_{\gamma \bar{\tau}}+\phi_{s} \overline{z^{\gamma}} z^{\tau}
$$

since

$$
\frac{\partial\left(\phi-s \phi_{s}\right)}{\partial s}=-s \phi_{s s}=0
$$

thus, $G_{\alpha \bar{\beta}}$ actually depends only on $z=\left(z^{1} \cdots z^{n}\right)$, i.e., $F$ comes from a Hermitian metric. 
Next we shall prove the necessity part of the theorem. Let $F=\sqrt{r \phi(t, s)}$ be a complex Landsberg metric, then we have

$$
2 G_{\gamma \bar{\tau}} \mathbb{G}_{v \mu}^{\gamma}=\left(G_{\mu \bar{\tau} ; \nu}+G_{v \bar{\tau} ; \mu}\right)-\left(\mathbb{G}_{\nu}^{\alpha} G_{\mu \bar{\tau} \alpha}+G_{\mu}^{\beta} G_{v \bar{\tau} \beta}\right)
$$

Using (2.1) and (2.7), after a long calculation, we have

$$
\begin{aligned}
4 G_{\gamma \bar{\tau}} \mathbb{G}_{v \mu}^{\gamma}= & \left.2\left(\phi-s \phi_{s}\right)\left[n_{1} \delta_{v \tau} \overline{z^{\mu}}+n_{1} \delta_{\mu \tau} \overline{z^{v}}-\frac{1}{r}\left(n_{1}-k_{2}\right) \overline{\langle z, v\rangle} \overline{\left(\overline{v^{\mu}}\right.} \delta_{v \tau}-\overline{v^{v}} \delta_{\mu \tau}\right)\right] \\
& +\frac{2 s}{r}\left[s^{2}(t-s) \phi_{s s} m_{3}-k_{4} n_{2}+\left(2 n_{1}-k_{2}\right) s \phi_{s s}\right] \overline{z^{\mu} v^{v} v^{\tau}} \\
& +\frac{2 s}{r}\left[s^{2}(t-s) \phi_{s s} m_{3}-k_{4} n_{2}+\left(2 n_{1}-k_{2}\right) s \phi_{s s}\right] \overline{z^{v} v^{\mu} v^{\tau}} \\
& +\left[2 k_{5}\left(s m_{3}+m_{1}+k_{3}\right)+2 s \phi_{s} n_{2}+4 k_{6} n_{1}\right] \overline{z^{\mu} z^{v}} z^{\tau} \\
& +\frac{2}{r}\left[-s(t-s) \phi_{s s}\left(s m_{3}+m_{1}+k_{3}\right)+k_{4} n_{2}-2 s \phi_{s s} n_{1}\right]\langle z, v\rangle \overline{z^{\mu} z^{v} v^{\tau}} \\
& +\frac{2 s}{r^{2}}\left[-s^{2}(t-s) \phi_{s s} m_{2}+k_{4} n_{2}-2 s \phi_{s s}\left(n_{1}-k_{2}\right)\right] \overline{\langle z, v\rangle \overline{v^{\mu} v^{v}} v^{\tau}} \\
& -\frac{2 s}{r}\left[k_{5} m_{3}+\phi_{s} n_{3}+\phi_{s s}\left(2 n_{1}-k_{2}\right)\right] \overline{\langle z, v\rangle} \overline{z^{v} v^{\mu}} z^{\tau} \\
& -\frac{2 s}{r}\left[k_{5} m_{3}+\phi_{s} n_{3}+\phi_{s s}\left(2 n_{1}-k_{2}\right)\right] \overline{\langle z, v\rangle} \overline{z^{\mu} v^{v}} z^{\tau} \\
& \left.+\frac{2 s}{r^{2}}\left[k_{5} m_{2}+\phi_{s} n_{2}+2 \phi_{s s}\left(n_{1}-k_{2}\right)\right] \overline{(z, v\rangle}\right)^{2} \overline{v^{\mu} v^{v}} z^{\tau},
\end{aligned}
$$

where we denote

$$
n_{3}=s \frac{\partial^{2} k_{2}}{\partial s^{2}}+3 \frac{\partial k_{2}}{\partial s}, \quad k_{4}=\phi-s \phi_{s}, \quad k_{5}=\phi+(t-s) \phi_{s}+s(t-s) \phi_{s s}, \quad k_{6}=\phi_{s}+s \phi_{s s} .
$$

Interchanging the indices $\mu$ and $v$ in (2.9), and then adding the obtained equality to (2.9), we get

$$
\begin{aligned}
G_{\mu \bar{\tau} ; v}+G_{v \bar{\tau} ; \mu}= & \left(\phi_{s}+\phi_{t}+s \phi_{s s}-s \phi_{s t}\right)\left(\delta_{\tau v} \overline{z^{\mu}}+\delta_{\mu \bar{\tau}} \overline{z^{v}}\right)-\frac{2 s}{r} \phi_{s s} \overline{\langle z, v\rangle}\left(\delta_{\mu \bar{\tau}} \overline{v^{v}}+\delta_{v \tau} \overline{v^{\mu}}\right) \\
& +2\left(s \phi_{s s t}+\phi_{s t}\right) \overline{z^{\mu} z^{v}} z^{\tau}+\frac{1}{r} s k_{7}\left(\overline{z^{\mu} v^{v}} v^{\tau}+\overline{z^{v} v^{\mu}} v^{\tau}\right) \\
& -\frac{2 s}{r} \phi_{s s t}\langle z, v\rangle \overline{z^{\mu} z^{v} v} v^{\tau}-\frac{1}{r} k_{7} \overline{\langle z, v\rangle}\left(\overline{z^{\mu} v^{v}} z^{\tau}+\overline{z^{v} v^{\mu}} z^{\tau}\right) \\
& +\frac{2}{r^{2}} s k_{8} \overline{\langle z, v\rangle} \overline{v^{\mu} v^{v}} v^{\tau}-\frac{2}{r^{2}}\left(k_{8}-\phi_{s s}\right)(\overline{\langle z, v\rangle})^{2} \overline{v^{\mu} v^{v}} z^{\tau}
\end{aligned}
$$

where we denote

$$
k_{7}=s \phi_{s s t}-s \phi_{s s s}-2 \phi_{s s}, \quad k_{8}=s \phi_{s s s}+2 \phi_{s s}
$$


Using (2.7), (2.8), and notice that $G_{\mu \bar{\tau} \alpha} v^{\alpha}=0, G_{\mu \bar{\tau} \alpha} v^{\bar{\tau}}=G_{\mu \alpha}=r \phi_{s s} s_{\alpha} s_{\mu}$, we obtain

$$
\begin{aligned}
2 \mathrm{G}_{\nu}^{\alpha} G_{\mu \bar{\tau} \alpha}+2 \mathrm{G}_{\mu}^{\beta} G_{v \bar{\tau} \beta}= & -s^{2}\left[(t-s)\left(m_{1}+k_{3}\right)+2 k_{2}\right]\left(\phi_{s s} \overline{z^{\mu}} \delta_{v \tau}+\phi_{s s} \overline{z^{v}} \delta_{\mu \bar{\tau}}\right) \\
& +\frac{s^{2}}{r}\left[(t-s)\left(m_{1}-k_{3}\right)+2 k_{2}\right]\left(\phi_{s s} \overline{\langle z, v\rangle} \overline{v^{\mu}} \delta_{v \tau}+\phi_{s s} \overline{\langle z, v\rangle} \overline{v^{v}} \delta_{\mu \bar{\tau}}\right) \\
& +\frac{s^{2}}{r}\left[2 k_{8} k_{2}+2 k_{9} k_{3}+\left(k_{9}+k_{10}\right)\left(m_{1}-k_{3}\right)\right]\left(\overline{z^{\mu} v^{v}} v^{\tau}+\overline{z^{v} v^{\mu}} v^{\tau}\right) \\
& +2\left[s k_{8} k_{2}+s k_{9}\left(m_{1}+k_{3}\right)\right] \overline{z^{\mu} z^{v}} z^{\tau} \\
& -\frac{2 s}{r}\left[\left(k_{8}-\phi_{s s}\right) k_{2}+k_{10}\left(m_{1}+k_{3}\right)\right]\langle z, v\rangle \overline{z^{\mu} z^{v}} v^{\tau} \\
& -\frac{2 s^{2}}{r^{2}}\left[\left(k_{8}+\phi_{s s}\right) k_{2}+k_{9}\left(m_{1}-k_{3}\right)\right] \overline{\langle z, v\rangle} \overline{v^{\mu} v^{v} v^{\tau}} \\
& -\frac{2 s}{r}\left(k_{8} k_{2}+k_{9} m_{1}\right) \overline{\langle z, v\rangle} \overline{z^{v} v^{\mu}} z^{\tau}-\frac{2 s}{r}\left(k_{8} k_{2}+k_{9} m_{1}\right) \overline{\langle z, v\rangle} \overline{z^{\mu} v^{v}} z^{\tau} \\
& +\frac{2 s}{r^{2}}\left[k_{8} k_{2}+k_{9}\left(m_{1}-k_{3}\right)\right](\overline{\langle z, v\rangle})^{2} \overline{v^{\mu} v^{v}} z^{\tau},
\end{aligned}
$$

where we denote

$$
k_{9}=(2 t-3 s) \phi_{s s}+s(t-s) \phi_{s s s}, \quad k_{10}=(t-2 s) \phi_{s s}+s(t-s) \phi_{s s s} .
$$

Substituting (3.3), (3.4) and (3.5) into (3.2), and comparing coefficients of the same types on both sides of the resulted equality, we obtain the following system of equations

$$
\left\{\begin{array}{l}
2 k_{4} n_{1}=2\left(\phi_{s}+s \phi_{s s}+\phi_{t}-s \phi_{s t}\right)+s^{2}(t-s) \phi_{s s}\left(m_{1}+k_{3}\right)+2 s^{2} \phi_{s s} k_{2} \\
2 k_{4}\left(n_{1}-k_{2}\right)=4 s \phi_{s s}+s^{2}(t-s) \phi_{s s}\left(m_{1}-k_{3}\right)+2 s^{2} \phi_{s s} k_{2} \\
2 s^{2}(t-s) \phi_{s s} m_{3}-2 k_{4} n_{2}+2\left(2 n_{1}-k_{2}\right) s \phi_{s s}=2 k_{7}-2 s k_{8} k_{2}-2 s k_{9} k_{3}-s\left(k_{9}+k_{10}\right)\left(m_{1}-k_{3}\right) \\
k_{5}\left(s m_{3}+m_{1}+k_{3}\right)+s \phi_{s} n_{2}+2 k_{6} n_{1}=2\left(s \phi_{s s t}+\phi_{s t}\right)-s k_{8} k_{2}-s k_{9}\left(m_{1}+k_{3}\right) \\
-s(t-s) \phi_{s s}\left(s m_{3}+m_{1}+k_{3}\right)+k_{4} n_{2}-2 s \phi_{s s} n_{1}=-2 s \phi_{s s t}+s\left(k_{8}-\phi_{s s}\right) k_{2}+s k_{10}\left(m_{1}+k_{3}\right) \\
-s^{2}(t-s) \phi_{s s} m_{2}+k_{4} n_{2}-2 s \phi_{s s}\left(n_{1}-k_{2}\right)=2 k_{8}+s\left(k_{8}+\phi_{s s}\right) k_{2}+s k_{9}\left(m_{1}-k_{3}\right) \\
k_{5} s m_{3}+s \phi_{s} n_{3}+s \phi_{s s}\left(2 n_{1}-k_{2}\right)=k_{7}-s k_{8} k_{2}-s k_{9} m_{1} \\
k_{5} s m_{2}+s \phi_{s} n_{2}+2 s \phi_{s s}\left(n_{1}-k_{2}\right)=-2\left(k_{8}-\phi_{s s}\right)-s k_{8} k_{2}-s k_{9}\left(m_{1}-k_{3}\right) .
\end{array}\right.
$$

The first equation of (3.7) minus the second equation of (3.7) yields

$$
s^{2}(t-s) \phi_{s s} k_{3}=k_{4} k_{2}-\left(\phi_{s}-s \phi_{s s}+\phi_{t}-s \phi_{s t}\right) .
$$

Taking the derivative with respect to the variable $s$, we obtain

$$
s^{2}(t-s) \phi_{s s} \frac{\partial k_{3}}{\partial s}=k_{4} \frac{\partial k_{2}}{\partial s}-s \phi_{s s} k_{2}+s \phi_{s s s}+s \phi_{s s t}-s k_{9} k_{3} .
$$

The third equation of (3.7) plus two times of the sixth equation of (3.7) yields

$$
s^{2}(t-s) \phi_{s s} \frac{\partial k_{3}}{\partial s}=2\left(k_{7}+2 k_{8}\right)-2 s k_{9} k_{3}
$$


Substituting (3.10) into the second equation of (3.7), one gets

$$
2 k_{4} \frac{\partial k_{2}}{\partial s}=4 \phi_{s s}+2\left(k_{7}+2 k_{8}\right)-2 s k_{9} k_{3}-s(t-s) \phi_{s s} k_{3}+2 s \phi_{s s} k_{2} .
$$

By (3.9)-(3.11), it follows that

$$
s(t-s) \phi_{s s} k_{3}=0 \text {. }
$$

Now it is easy to see that the Eq. (3.12) holds if and only if either

$$
\phi_{s S}=0,
$$

or

$$
k_{3}=0
$$

holds.

If (3.13) holds, we immediately have

$$
\phi(t, s)=a_{0}(t)+a_{1}(t) s,
$$

where $a_{0}(t)$ and $a_{1}(t)$ are two smooth real-valued functions satisfying $a_{0}(t)>0$ and $a_{0}(t)+$ $t a_{1}(t)>0$.

If (3.14) holds, according to (2.5), we have

$$
\phi_{s s}+\phi_{s t}=\frac{\phi_{s}\left(\phi_{t}+\phi_{s}\right)}{\phi} .
$$

Substituting (3.15) into (2.4), we get

$$
k_{2}=\frac{\phi_{t}+\phi_{s}}{\phi k_{1}}\left\{\left(\phi-s \phi_{s}\right)\left[\phi+(t-s) \phi_{s}\right]+s(t-s) \phi \phi_{s s}\right\}=\frac{\phi_{t}+\phi_{s}}{\phi k_{1}} k_{1}=\frac{\phi_{t}+\phi_{s}}{\phi} .
$$

Thus

$$
\frac{\partial k_{2}}{\partial s}=\frac{1}{\phi^{2}}\left[\phi\left(\phi_{s t}+\phi_{s s}\right)-\phi_{s}\left(\phi_{t}+\phi_{s}\right)\right]=\frac{1}{\phi} k_{1} k_{3}=0 .
$$

Substituting (3.14) and (3.17) into the second and eighth equations of (3.7), respectively, we obtain

$$
\left\{\begin{array}{l}
s k_{2}=-2, \\
2 \phi_{s s}=\left(s k_{2}+2\right) k_{8},
\end{array}\right.
$$

from which we get (3.13). This completes the proof of the Theorem.

Remark 3.1. Theorem 3.1 implies as far as that unitary invariant strongly pseudoconvex complex metrics are concerned, $F=\sqrt{r \phi(t, s)}$ is a complex Landsberg metric if and only if it comes from a unitary invariant Hermitian metric. Thus there is no unicorn metric among unitary invariant strongly pseudoconvex complex Finsler metrics. 


\section{Acknowledgments}

This work was supported by NCET (Grant No.13-0510), NSFC (Grant Nos. 11271304, 11171277, 11571288, 11461064) and the Fujian Province Natural Science Funds for Distinguished Young Scholar (Grant No. 2013J06001).

\section{References}

[1] M. Abate, G. Patrizio. Finsler metrics-A global approach with applications to geometric function theory. Lect. Notes in Math., Springer-Verlag, Berlin, Heidelberg, 1994, 1591.

[2] M. Matsumoto. Remarks on Berwald and Landsberg spaces, in Finsler Geometry. Contemp. Math., 1996 196: 79-82.

[3] D. Bao. On two curvature-driven problems in RiemannFinsler geometry. Finsler geometry, Sapporo 2005-in memory of Makoto Matsumoto, 1971, Adv. Stud. Pure Math., 48, Math. Soc. Japan, Tokyo, 2007.

[4] Z.I. Szabó. All regular Landsberg metrics are Berwald. Ann. Global Anal. Geom. 2008, 34(4): 381-386.

[5] Z.I. Szabó. Correction to all regular Landsberg metrics are Berwald. Ann. Global Anal. Geom. 2009, 35(3): 227-230.

[6] G.S. Asanov. Finsleroid-Finsler space with Berwald and Landsberg conditions. E-print, arXiv: math/0603472, 2006.

[7] G.S. Asanov. Finsleroid-Finsler space and spray coefficients. E-print arXiv: math/0604526, 2006.

[8] Z. Shen. On a class of Landsberg metrics in Finsler geometry. Can. J. Math. 2009, 61(6): 13571374.

[9] X.H. Mo, L.F. Zhou. The curvatures of spherically symmetric Finsler metrics in $R^{n}$. E-print arXiv: 1202. 4543 [math.DG], 2014.

[10] N. Aldea, G. Munteanu. On complex Landsberg and Berwald spaces. J. Geom. Phys. 2012, 62(2): 368-380.

[11] G. Munteanu. Complex spaces in Finsler. Lagrange and Hamilton Geometries, Kluwer Academic Publishers, 2004.

[12] C.P. Zhong. On unitary invariant strongly pseudoconvex complex Finsler metrics. Differ. Geom. Appl. 2015, 40: 159-186.

[13] H.C. Xia, C.P. Zhong. A classification of unitary invariant weakly complex Berwald metrics of constant holomorphic curvature, Differ. Geom. Appl. 2015, 43: 1-20. 